\section{A) Check for updates}

Cite this: Food Funct., 2019, 10, 1540

\title{
Protection of hepatocyte mitochondrial function by blueberry juice and probiotics via SIRT1 regulation in non-alcoholic fatty liver disease $\uparrow$
}

\author{
Tingting Ren, $\xi^{\mathrm{a}, \mathrm{b}}$ Lili Zhu, $\xi^{\mathrm{c}}$ Yanyan Shen, ${ }^{\mathrm{d}}$ Qiuju Mou, ${ }^{\mathrm{e}}$ Tao $\operatorname{Lin}^{\mathrm{f}}$ and \\ Hongchao Feng (D) *9
}

\begin{abstract}
Mitochondrial dysfunction has been implicated in the pathogenesis of nonalcoholic fatty liver disease (NAFLD). Our previous study has firstly reported that blueberry juice and probiotics (BP) effectively protect liver function in NAFLD. However, the role of BP in hepatic mitochondria is unknown. Here, we aimed to investigate the effects and mechanisms of $\mathrm{BP}$ on the mitochondrial function and oxidative stress of rats with NAFLD. The NAFLD rat models were established and treated with BP and SIRT1 siRNA. The mitochondrial ultrastructure was analyzed by electron microscopy, reactive oxygen species (ROS) was detected by immunofluorescence, and biomarkers of mitochondrial function and oxidative stress were examined via quantitative reverse transcription-PCR, western blot, and immunohistochemistry. Results revealed that BP significantly reversed the NAFLD-induced hepatic mitochondrial damage, mitochondrial swelling, and hepatic necrosis. In particular, BP significantly restored the mitochondrial respiratory function of NAFLD rats by decreasing state 4 and 3 respiration rates, by increasing the respiration control ratio (RCR) and the ADP/O ratio, and by enhancing ATP, ADP, AMP, and EC syntheses. Moreover, BP reduced mitochondrial oxidative stress in NAFLD by decreasing the MDA level, elevating the GSH and SOD levels, and suppressing the ROS activity. Importantly, SIRT1 deficiency significantly abolished the effects of BP on the mitochondria and oxidative stress. Furthermore, BP reversed the decline of PGC-1 $\alpha$ expression induced by NAFLD, while SIRT1 silencing significantly suppressed the effects of BP on PGC-1 $\alpha$. In conclusion, BP might effectively protect rats against mitochondrial dysfunction during NAFLD as potential ingredients of functional food, by modulating the SIRT1 expression. Potential endogenous modulators of NAFLD pathogenesis may ultimately provide novel tools for therapeutic intervention.
\end{abstract}

Received 22nd November 2018 Accepted 28th January 2019

DOI: $10.1039 / \mathrm{c} 8 \mathrm{fo} 02298 \mathrm{~d}$

rsc.li/food-function range of diseases, from simple hepatic steatosis to non-alcoholic steatohepatitis (NASH) with enhanced inflammation and fibrosis, to cirrhosis of the liver. ${ }^{2}$ Various risk factors can lead to NAFLD, for instance, hypertension, insulin resistance, central adiposity, and metabolic syndrome. ${ }^{3}$ The histological characteristic of NAFLD is the accumulation of lipids in hepatocytes. ${ }^{4}$ The excessive fat in the hepatocytes results in the imbalance of lipid metabolism and triggers lipotoxicity and oxidative stress, which facilitates mitochondrial dysfunction via various signaling pathways. ${ }^{5,6}$ Therefore, oxidative stress and mitochondrial dysfunction are of vital importance for the development of NAFLD.

Berries are one of the most beneficial dietary sources, due to their unique chemical and biological properties, including, trace elements, flavonoids, vitamins, and particularly, polyphenols, for instance, anthocyanins, which exhibit anti-oxidative properties. $^{7}$ It has been shown that blueberries play an important role in hepatic protection and hepatic fibrosis reversal. ${ }^{8}$ Moreover, an increasing number of studies have revealed that 
probiotics act as a complementary therapeutic method in NAFLD. ${ }^{9}$ However, little is known about the impacts of blueberry juice combined with probiotics (BP) on NAFLD. Our previous study showed that BP could effectively improve NAFLD by regulating a PPAR- $\alpha$-mediated SREBP-1c/PNPLA-3 pathway. ${ }^{10}$ Nevertheless, the mechanisms of how BP influences NAFLD are still poorly understood.

Silence information regulatory factor 1 (SIRT1) is an NADdependent histone deacetylase that plays a vital role in the regulation of gene transcription, apoptosis, cell cycle, metabolism, and development. SIRT1 has been shown to increase cell cycle arrest and promote resistance to oxidative stress. ${ }^{11}$ In particular, as an important molecule involved in the rapid regulation of enzyme activity, SIRT1 plays a deleterious role in negatively regulating the initiation and progression of fatty liver diseases. ${ }^{12}$ In addition, peroxisome proliferator-activated receptor alpha co-activator (PGC-1 $\alpha$ ), a cytokine, which is downstream of SIRT1, has been reported to upregulate mitochondrial biogenesis and function. ${ }^{13}$ However, whether BP improve NAFLD through SIRT1/PGC-1 $\alpha$-mediated mitochondrial regulation is still unknown.

In this study, we aimed to study the effects of BP on oxidative stress and mitochondria during NAFLD. NAFLD rat models were established and treated with $\mathrm{BP}$, and their liver cell mitochondria were observed. Moreover, we focused on SIRT1 and PGC- $1 \alpha$ to elucidate the molecular mechanisms involved in the regulation of production and function of mitochondria by BP.

\section{Materials and methods}

\section{Ethics}

All the protocols in this study were approved by the animal care and ethical committee of the Affiliated Hospital of Guizhou Medical University. All animal handling procedures were performed according to the Guide for the Care and Use of Laboratory Animals of the National Institutes of Health and followed the guidelines of the Animal Welfare Act.

\section{Preparation of BP}

Blueberries were obtained from a Majiang blueberry plant (Guiyang, China). Blueberry juice was obtained as described in a previous study. ${ }^{14}$ Briefly, $1 \mathrm{~kg}$ of blueberry blend was thawed at $4{ }^{\circ} \mathrm{C}$ for $8 \mathrm{~h}$ and crushed using a Braun Global hand blender (MR300; De'Longhi Kenwood A.P.A Ltd, Hong Kong, China). The milled blueberries were pressed under the maximum pressure of $0.9 \mathrm{MPa}$ in a bag press mod. The prepared blueberry juice was given to rats immediately. The main composition of blueberry juice was detected as described in the previous study. ${ }^{15}$ As shown in our previous study, ${ }^{16}$ the anthocyanin concentration in blueberry juice was $0.98 \pm 0.07 \mathrm{mg} \mathrm{mL} \mathrm{m}^{-1}$. Dry probiotic tablets mixed with the probiotic species of Bifidobacterium infantis, Bifidobacterium animalis, and Lactobacillus acidophilus were obtained from the China General Microbiological Culture Collection Center (Beijing,
China). Dried probiotic tablets (each tablet contained $5 \times 10^{6}$ CFU $\mathrm{mL}^{-1}$ bacteria) were ground into powder, and $10 \mathrm{~mL}$ of blueberry juice was added with a piece of probiotics and incubated at $4{ }^{\circ} \mathrm{C}$. The live bacteria in the juice were monitored as follows. After incubation for $6 \mathrm{~h}, 12 \mathrm{~h}$, and $24 \mathrm{~h}, 0.01 \mathrm{~mL}$ of the blueberry probiotic mixture were taken and the total number of colonies was calculated directly under the microscope. The probiotic survival rate $(\%)=\lg$ N1/g N0 $\times 100 \%$ (N1: the number of live bacteria $\left(\mathrm{CFU} \mathrm{mL} \mathrm{m}^{-1}\right.$ ) after treatment; No: the initial quantity of live bacteria $\left(\mathrm{CFU} \mathrm{mL}^{-1}\right)$ ). The number of live bacteria at $6 \mathrm{~h}, 12 \mathrm{~h}$, and $24 \mathrm{~h}$ was $5.9 \times 10^{7}$, $6.3 \times 10^{7}$, and $5.2 \times 10^{8} \mathrm{CFU} \mathrm{mL}^{-1}$, respectively. BP with a concentration of $10^{8} \mathrm{CFU} \mathrm{\textrm {mL } ^ { - 1 }}$ probiotics was used for further study.

\section{Establishment of the NAFLD rat model}

Sprague-Dawley rats (weight 200-250 g) were purchased from the Animal Center of Guiyang Medical College (Approval Number SCXK (Guizhou) 2002-0001, Guiyang, China). Rats of the control group received a normal diet and rats of model groups were fed with a high-fat diet for two months. The highfat diet was prepared as described in a previous study. ${ }^{1}$ The control diet was composed of $4.3 \%$ fat $(10 \%$ metabolizable energy), and the high-fat diet was composed of $35 \%$ fat $(31.6 \%$ saturated fat and $3.2 \%$ unsaturated fat and $57 \%$ metabolizable energy). The model was evaluated by histopathological detection in two rats and the 48 remaining rats were randomly divided into six groups (eight animals per group).

\section{SIRT1 SiRNA}

The pshRNA-H1-Luc lentivector was provided by System Biosciences (Mountain View, USA). The specific SIRT1 siRNA sequence (5'-CAGGTTGCAGGAATCCAAAG-3') and the control sequence (5'-TTCTCCGAACGTGTCACGT-3') were synthesized and cloned into the lentivector. Lentiviruses carrying siRNA sequences were generated and harvested in 293T producer cells with a pPACK Packaging Plasmid Mix (System Biosciences, Shanghai, China) and Lipofectamine ${ }^{\mathrm{TM}} 2000$ (Thermo Fisher Scientific, Waltham, MA, USA). The lentiviral supernatant was collected and the titer was calculated with diluted lentivirus after transfection for $48 \mathrm{~h}$. NAFLD mice were intra-articularly injected with $100 \mu \mathrm{L}$ supernatants containing the lentivirus-carrying SIRT1 siRNA $\left(1 \times 10^{8}\right.$ virus titer per injection, two times per day) for 10 days.

\section{Treatment with blueberry and probiotics}

After confirming the model and the siRNA effect, the blueberry groups were fed with blueberry juice (1.5 mL per $100 \mathrm{~g}$ weight) once a day, for 10 days. In addition, the BP groups were gavaged with blueberry juice $(1.5 \mathrm{~mL}$ per $100 \mathrm{~g}$ weight) and probiotics (the concentrations of probiotics were $250 \mathrm{mg}$ per $100 \mathrm{~mL}$ and $20 \mathrm{~mL}$ per $100 \mathrm{~g}$ ) once a day, for 10 days.

\section{Evaluation of the mitochondrial ultrastructure}

Animals were anesthetized with chloralose (40 $\mathrm{mg} \mathrm{kg}^{-1}, \mathrm{SC}$ ) and sacrificed. Liver tissues were collected and fixed with $2.5 \%$ 
glutaraldehyde on ice. After washing with $0.1 \mathrm{M}$ phosphate buffered solution (PBS), samples were fixed for $2 \mathrm{~h}$ in $1 \%$ osmic acid solution. Tissues were dehydrated in a cold graded ethanol series $(30 \%, 50 \%$ and $70 \%)$ for $10 \mathrm{~min}$ at $4{ }^{\circ} \mathrm{C}$, and then they were dehydrated in acetone solution ( $80 \%$ and $90 \%$ ) at $20{ }^{\circ} \mathrm{C}$ for $10 \mathrm{~min}$, and three times in $100 \%$ acetone solution for 15 min. After embedding in Epon812 overnight, samples were cut into semi-thin tissue sections of 100-120 nm. Sections were stained with $1 \%$ toluidine blue for $5 \mathrm{~min}$ and blocked with a rubber. The selected areas were cut at a thickness of 40-60 $\mathrm{nm}$ and stained for $30 \mathrm{~min}$ using uranyl acetate. The sections were examined using a JEM 1200EX transmission electron microscope (JEOL, Tokyo, Japan).

\section{Preparation of hepatic mitochondria}

The liver tissues from the same part of the rats were taken and washed with pre-cooled PBS, and then treated with precooled sucrose buffer $(1: 4)$. After fully homogenizing using a homogenizer in an ice bath, samples were centrifuged at $2500 \mathrm{rpm}$ at $4^{\circ} \mathrm{C}$ for $10 \mathrm{~min}$. Precipitates were removed and supernatants were centrifuged at $13000 \mathrm{rpm}$ at $4{ }^{\circ} \mathrm{C}$ for $10 \mathrm{~min}$, and the obtained precipitate was liver mitochondria. The protein content was determined by the Bradford method using $0.01 \mathrm{~mol} \mathrm{~L}^{-1}$ tris-HC1 buffer containing $0.01 \mathrm{~mol} \mathrm{~L}^{-1}$ EDTA.

\section{Determination of the degree of liver mitochondrial swelling}

One milligram of hepatic mitochondria proteins was added to $1 \mathrm{~mL}$ swelling test solution (contains $0.25 \mathrm{~mol} \mathrm{~L}^{-1}$ sucrose, $5 \times$ $10^{-3} \mathrm{~mol} \mathrm{~L}^{-1} \mathrm{KH}_{2} \mathrm{PO}_{4}, 3 \times 10^{-3} \mathrm{~mol} \mathrm{~L}^{-1}$ sodium succinate, and $3 \times 10^{-4} \mathrm{~mol} \mathrm{~L}^{-1} \mathrm{CaCl}_{2}$ ). The absorbance value of the hepatic mitochondrial suspension at $520 \mathrm{~nm}$ was measured at $25{ }^{\circ} \mathrm{C}$, after incubating for $0 \mathrm{~min}\left(A_{0}\right)$ and $20 \mathrm{~min}\left(A_{20}\right)$, respectively. The value $\Delta A=(a 0-a 20)$ indicates the swelling degree of liver mitochondria. Larger $\Delta A$ indicates stronger buffer capacity of liver mitochondria to calcium ions and better structural integrity of the mitochondria.

\section{Detection of the liver necrosis area}

Liver tissue was washed with PBS and fixed in 4\% paraformaldehyde, and then it was prepared for conventional paraffin embedding, cut into 4-micron sections, and stained with hematoxylin and eosin. Under $10 \times 20$ times visual field, 5 visual fields were randomly selected from each sample. The area of hepatic necrosis was measured using the Olympus BX41 image system (Olympus Company, Tokyo, Japan).

\section{Detection of the mitochondrial respiratory function}

The respiratory control ratio (RCR) refers to the ratio of the state 3 respiratory rate (when ADP is activated) to the state 4 respiratory rate (when mitochondrial succinic acid is activated after ADP is exhausted). The respiration rate of state 3 and state 4 was measured by the Clark oxygen electrode technique. The total volume of the reaction system was $3 \mathrm{~mL}, \mathrm{pH}$ : 7.35-7.45, including $0.01 \mathrm{~mol} \mathrm{~L}^{-1} \mathrm{KC} 1,5 \mathrm{mmol} \mathrm{L}{ }^{-1} \mathrm{MgC}_{2}$,
$0.2 \mathrm{~mol} \mathrm{~L}^{-1}$ succinic acid, $0.01 \mathrm{~mol} \mathrm{~L}^{-1}$ Tris-HC1, $0.25 \mathrm{mmol}$ $\mathrm{L}^{-1}$ sucrose, $5 \mathrm{mmol} \mathrm{L}{ }^{-1} \mathrm{KH}_{2} \mathrm{PO}_{4}, 0.33 \mathrm{mmol} \mathrm{L}{ }^{-1} \mathrm{ADP}$, and $1 \mathrm{~g} \mathrm{~L}^{-1}$ mitochondrial protein. The RCR and phosphorus/oxidation $(\mathrm{ADP} / \mathrm{O})$ was calculated.

\section{Evaluation of reactive oxygen species (ROS)}

Liver tissues were placed in cold PBS, snipped, and added with trypsin, and digested by oscillations at $37{ }^{\circ} \mathrm{C}$ for $20 \mathrm{~min}$. After discarding the supernatants, samples were digested by IV collagenase at $37^{\circ} \mathrm{C}$ for $40 \mathrm{~min}$ and filtered by 120 and 200 meshes. The cell suspension was centrifuged at $800 \mathrm{rpm}$ for $3 \mathrm{~min}$ at $4{ }^{\circ} \mathrm{C}$ and repeatedly centrifuged 3 times. After cell collection, the cells were incubated with a fluorescent probe DCFH-DA (to detect intracellular ROS, $10 \mu \mathrm{mol} \mathrm{L}{ }^{-1}$, with an excitation wavelength of $488 \mathrm{~nm}$ and an emission wavelength of $530 \mathrm{~nm}$ ) for $30 \mathrm{~min}$, and then washed with PBS 3 times to fully remove the fluorescent probe that did not enter the cells. The cells were added with $2 \mathrm{~mL}$ PBS and the fluorescence intensity of each group was measured by fluorescence microscopy with a full-wavelength scanning multifunctional reading device.

\section{Immunohistochemical staining}

Liver tissues were fixed in $4 \%$ paraformaldehyde and embedded in the OCT compound (Sakura, USA), followed by cutting into $6 \mu \mathrm{m}$ thick sections. The sections were deparaffinized, followed by antigen retrieval using a target-retrieval solution (Dako) in a steamer for $45 \mathrm{~min}$, and incubated with primary antibodies against SIRT1 (Abcam \#104833; $7: 1000$ ) and PGC-1 $\alpha$ (Abcam \#54481; 1 : 500). Then, the sections were incubated with a goat anti-rabbit secondary antibody (BA1081). The results were observed using an Olympus BX41 and the Mean Optical Density was analyzed using an MIAS (MIAS 2000, Olympus, Tokyo, Japan) for five random visual fields of each section under $400 \times$.

\section{Western blot}

The total protein of liver tissues was extracted and the concentration was measured using a BCA assay kit (Pierce Biotechnology, Inc., Rockford, IL, USA). Protein samples $(30 \mu \mathrm{g})$ from each group were loaded on 10\% SDS-PAGE gel and transferred to polyvinylidene fluoride membranes (Millipore, Bedford, MA, USA). Membranes were then treated with primary antibodies against SIRT1 $(1: 2000)$, PGC-1 $\alpha$ (1:1000), and GAPDH (PB0141; $1: 1000)$ overnight at $4{ }^{\circ} \mathrm{C}$, followed by washing and incubation with the goat anti-rabbit IgG-HRP secondary antibody for 2 hours. Protein bands were detected with an enhanced chemiluminescence reagent (Thermo Fisher Scientific, Waltham, MA) and photographed using a Chemi Doc MP system (Bio-Rad, Hercules, USA), and the optical density was analyzed using Image J software.

\section{Quantitative reverse transcription-PCR (qRT-PCR)}

Total RNA was extracted from hepatic tissues using Trizol, and cDNA was generated according to the manuals using the 
QuantiTect Reverse Transcription Kit (Qiagen). The SYBR Premix Ex Taq ${ }^{\mathrm{TM}}$ (TaKaRa, Tokyo, Japan) was used to quantify the relative mRNA levels of SIRT1 and PGC- $1 \alpha$ on an ABI onestep fast thermocycler (Applied Biosystems, Paisley, UK). The PCR protocol was as follows: 1 cycle of $95{ }^{\circ} \mathrm{C}$ for $10 \mathrm{~min}$, 40 cycles of $95{ }^{\circ} \mathrm{C}$ for $5 \mathrm{~s}$, and annealing and extension at $55-58{ }^{\circ} \mathrm{C}$ for $30 \mathrm{~s}$. The mRNA level of GAPDH was used as an internal reference. The primers are shown in Table S1. $\dagger$ Data were analyzed using the $2^{-\Delta \Delta C T}$ method.

\section{Statistical analysis}

The data in this study were shown as mean \pm standard deviation (SD). Comparisons between two groups were analyzed by two-tailed Student's $t$-test and $P<0.05$ was considered a statistically significant difference.

\section{Results}

\section{BP restricts the buildup of damaged mitochondria in NAFLD}

To investigate the impact of BP on the mitochondrial ultrastructure, we used EM to directly assess the liver mitochondrial integrity and state in rat liver (Fig. 1A). In normal rats, the liver mitochondria displayed a normal size, intact membrane, and a clear mitochondrial crista. However, the mitochondria in NAFLD rats were significantly swollen, possessed a ruptured and dissolved mitochondrial membrane, harbored a fragmentary mitochondrial crista, and decreased in total numbers. After blueberry or BP treatment in NAFLD rats, the mitochondria showed a reduced swollen size, and a nearly intact membrane and crista, suggesting that blueberry and BP imparted protective effects on the mitochondrial structure during NAFLD. Moreover, hepatic mitochondrial swelling and necrotic area were significantly increased in the NAFLD rats compared to those in the normal rats, while blueberry and BP significantly restored the swelling and necrotic area to nearly the normal level (Fig. 1B and C). Notably, blueberry and BP significantly reversed the decreased mitochondrial respiratory function in NAFLD rats compared to the normal rats, by decreasing the state 4 and 3 respiration rates, and increasing the respiration control ratio ( $\mathrm{RCR}$ ) and the $\mathrm{ADP} / \mathrm{O}$ ratio (Fig. 1D, E, F and G). Moreover, the ATP, ADP, AMP, and EC synthesis by mitochondria was significantly inhibited in the NAFLD rats compared to the normal rats, while blueberry and BP significantly promoted their synthesis by mitochondria (Fig. 1H). Furthermore, the activities of mitochondrial CCO and SDH were significantly decreased in the NAFLD rats compared to the normal rats, while blueberry and $\mathrm{BP}$ significantly induced the activation of CCO and SDH (Fig. 1I and J).

\section{BP treatment reduced mitochondrial oxidative stress during NAFLD}

The mitochondrial MDA level was significantly elevated, while the levels of GSH and SOD were reduced during NAFLD compared with that in the normal rats, whereas, treatment with blueberry and BP restored the MDA, GSH, and SOD to their normal levels (Fig. 2A, B and C). The hepatic mitochondria from NAFLD rats also exhibited elevated mRNA and protein expression levels of GSH and SOD after treatment with blueberry or BP, out of which BP was more effective (Fig. 2D and E). Importantly, immunofluorescence revealed that the ROS activity was triggered by the NAFLD, whereas both blueberry and BP significantly suppressed the ROS expression; however, BP showed a more significant effect (Fig. 2F). These results suggested that blueberry and $\mathrm{BP}$ can both reduce mitochondrial oxidative stress during NAFLD, and that the anti-oxidative stress function of BP is stronger than that of blueberry.

\section{BP restored the decreased level of SIRT1 in NAFLD}

The mRNA and protein expression of SIRT1 in the NAFLD model group was significantly decreased compared to that in the normal group (Fig. 3A and B). After blueberry or BP treatment, the expression level of SIRT1 was significantly increased in NAFLD rats. Moreover, the NAFLD rats treated with BP expressed a significantly higher level of SIRT1 compared to the NAFLD rats treated with blueberry alone.

\section{SIRT1 deficiency triggered mitochondrial dysfunction}

To determine the role of SIRT1 on the mitochondrial function, we established the SIRT1 knockdown rats by treating with the lentivirus carrying SIRT1 siRNA. As shown in Fig. $\mathrm{S} 1, \dagger$ the mRNA and protein levels of SIRT1 were significantly decreased in the liver tissues of normal and BP pretreated NAFLD rats after injection with SIRT1 siRNA, suggesting that the SIRT1-silenced model rats were successfully established.

As expected, the degree of increase in hepatic mitochondrial swelling and hepatic necrosis of the healthy rats was comparable to that of the NAFLD rats, after SIRT1 knockdown (Fig. S2A and B†). With SIRT1 siRNA treatment in normal rats, state 4 and 3 respiration rates were significantly decreased, and the RCR and ADP/O ratio was increased to levels comparable to those of the NAFLD group (Fig. S2C, D, E, and $\mathrm{F}^{\dagger}$ ). Moreover, SIRT1 silencing significantly inhibited the synthesis of ATP, ADP, AMP, and EC (Fig. S2G $\dagger$ ), and the activities of $\mathrm{CCO}$ and $\mathrm{SDH}$ (Fig. $\mathrm{S} 2 \mathrm{H}$ and $\mathrm{I} \dagger$ ) in the mitochondria of normal rats. Similarly, SIRT1 silencing significantly altered the hepatic content of oxidative stress markers in the normal rats, including an increased MDA level and decreased GSH and SOD levels (Fig. S3†).

\section{SIRT1 was indispensable for the mitochondrial protection mediated by BP}

We next examined whether SIRT1 was involved in the effects of BP on NAFLD. SIRT1 deficiency significantly suppressed the protective function of $\mathrm{BP}$ in regulating the mitochondrial structure, by increasing the swelling and damaging the mitochondrial membrane and crista in NAFLD rats (Fig. 4A, B and C). Moreover, SIRT1 silencing completely abolished the protection of $\mathrm{BP}$ in mitochondrial respiration, including 
A
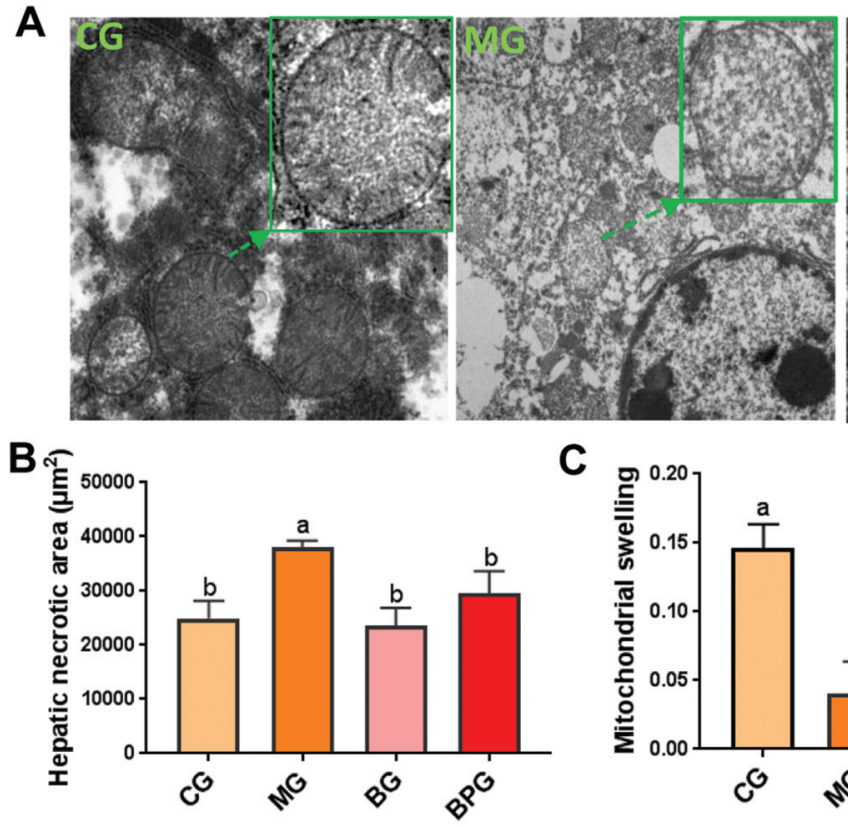

C

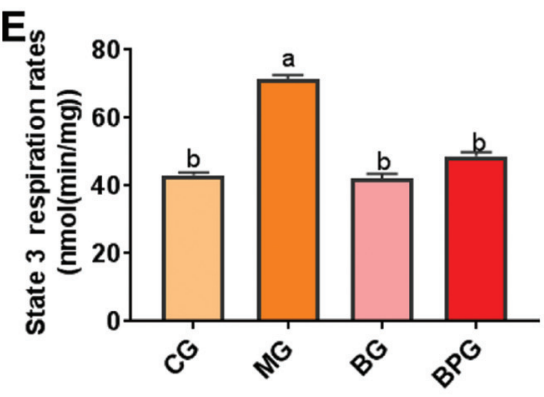

$F$

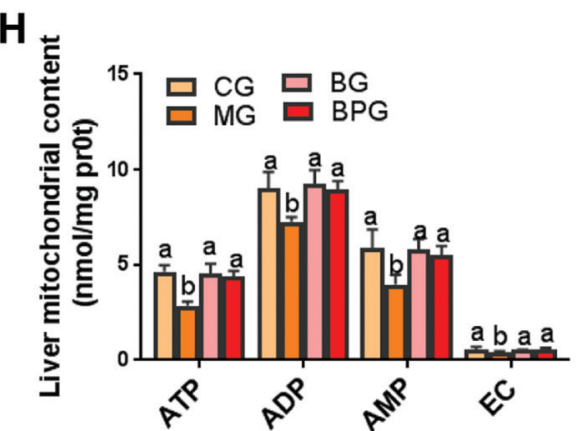

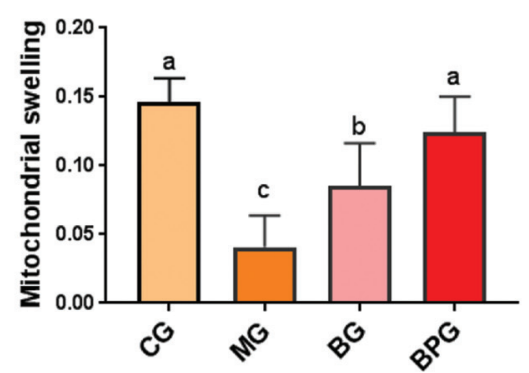
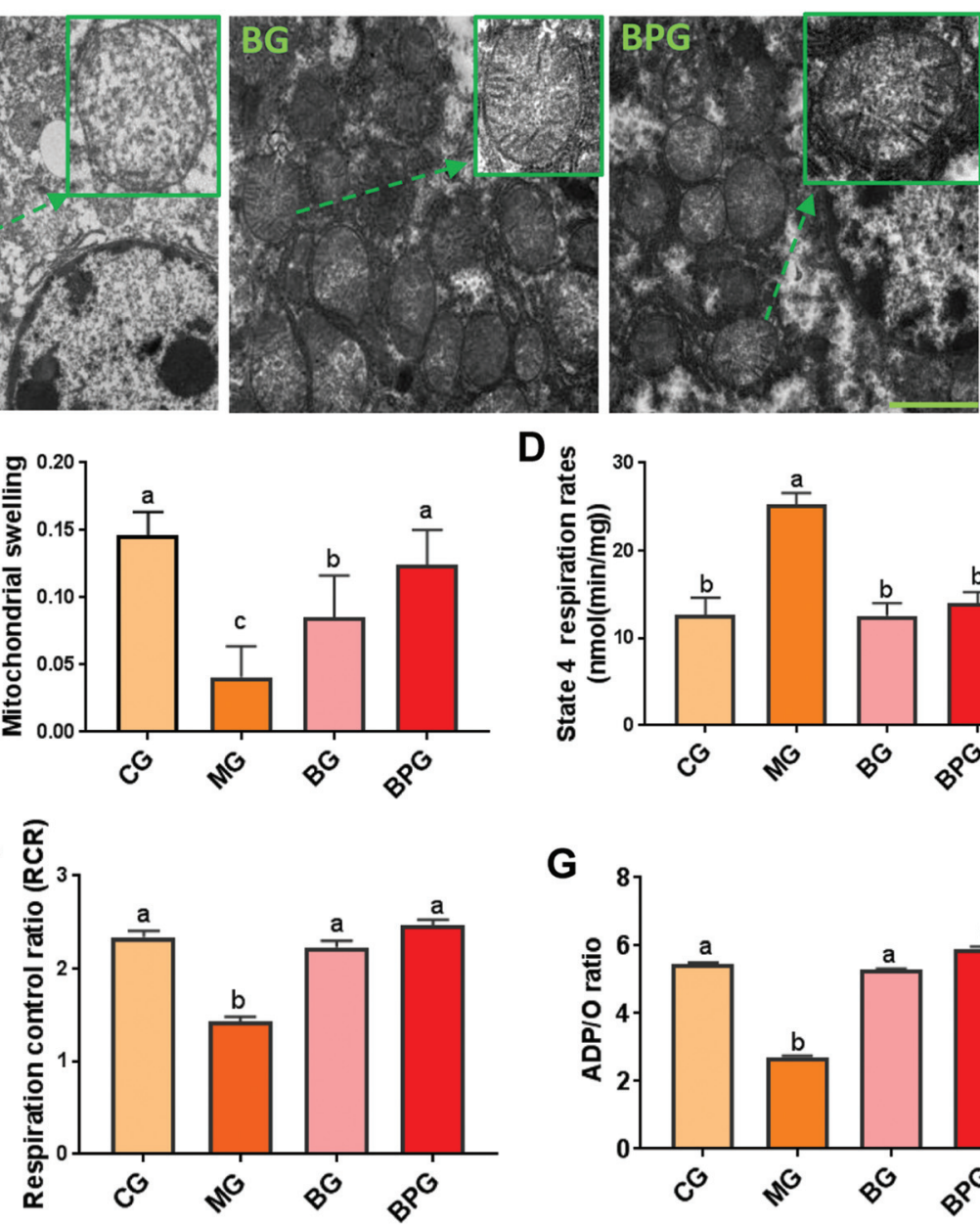

D

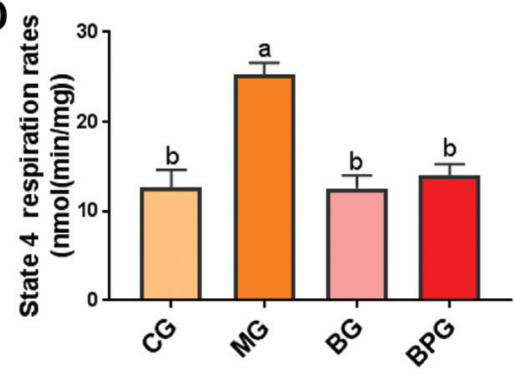

G

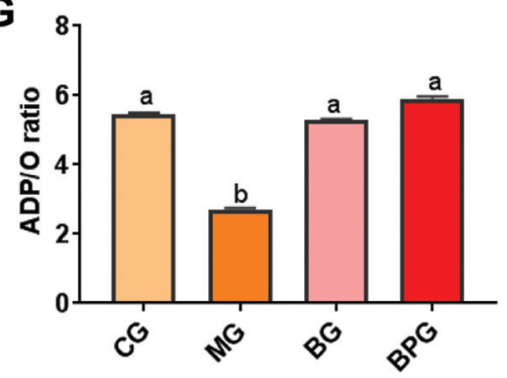

I
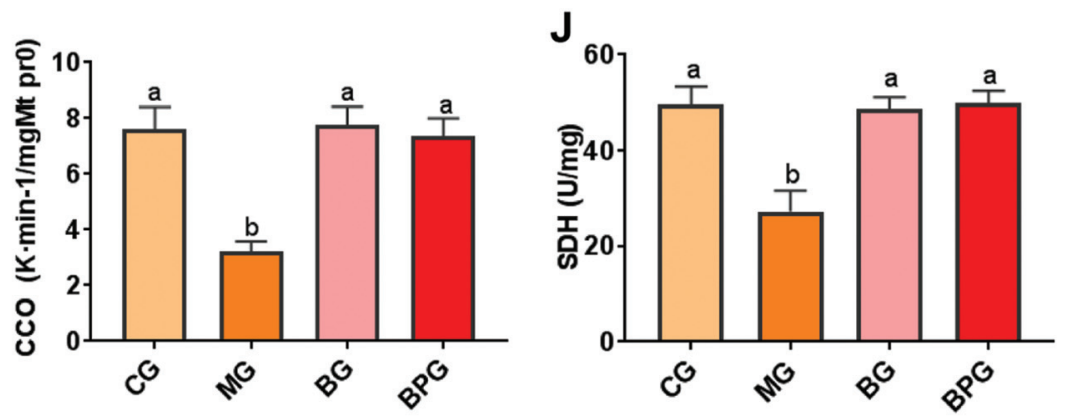

Fig. 1 BP restricts the buildup of damaged mitochondria in NAFLD. (A) Electron micrographs of mitochondria in different groups. Scale bars: $2 \mu$ m. (B) The area of hepatic necrosis was measured by a section of liver tissue using the Olympus BX41 image system. (C) The mitochondrial swelling degree was measured by the absorbance value of the hepatic mitochondrial suspension at $520 \mathrm{~nm}$. (D, E, F, and G) The effect of BP on the mitochondrial respiratory function was evaluated by state 4 and 3 respiration rates, RCR, and the ADP/O ratio. (H) Mitochondrial synthesis functions of ATP, ADP, AMP, and EC. (I and J) Mitochondrial function biomarkers were detected using a biochemical marker kit. CG, normal rats; MG, NAFLD model rats; and BG, model rats were treated with blueberry juice; BPG, model rats were treated with blueberries and probiotics. Different lowercase letters $(a, b, c$, and $d)$ represent significant differences $(P<0.05$, one way ANOVA, $n=8)$.

enhanced state 4 and 3 respiration rates, and decreased RCR and the ADP/O ratio (Fig. 4D, E, F, and G). With SIRT1 knockdown, BP also failed to promote the activation of CCO and SDH (Fig. 4H and I), and the synthesis of ATP, ADP, AMP, and EC (Fig. 4J).
SIRT1 was indispensable for the anti-oxidative stress properties of BP

The mitochondrial activity of MDA from NAFLD rats was significantly decreased, and the GSH and SOD levels were 

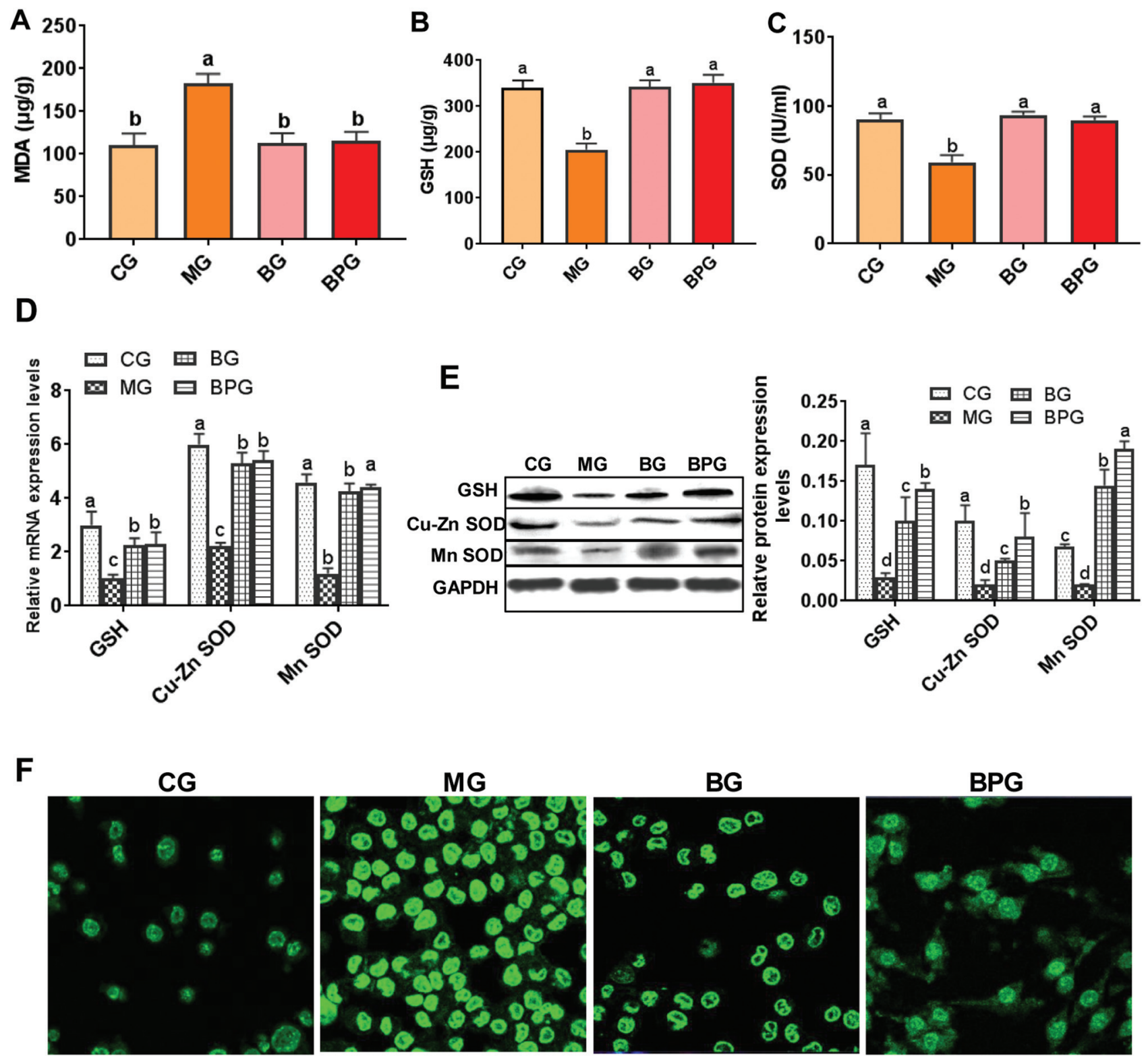

Fig. 2 BP reduced mitochondrial oxidative stress in NAFLD. (A, B and C) Oxidative stress biomarkers were detected using a biochemical marker kit. ( $D$ and E) Expression of SOD and GSH was measured by quantitative reverse transcription-PCR and western blot. (F) Reactive oxygen species (ROS) in the liver was detected by immunofluorescence $(x 400)$. Different lowercase letters $(a, b, c$, and d) represent significant differences $(P<0.05$, one way ANOVA, $n=8$ ).

increased after BP treatment, while SIRT1 siRNA significantly reversed the aforementioned changes (Fig. 5A, B, and $\mathrm{C}$ ). SIRT1 silencing significantly reduced the GSH and SOD mRNA levels in BP-treated NAFLD rats to the levels comparable to those of untreated NAFLD rats (Fig. 5D). The protein levels of GSH and SOD in NAFLD rats after BP and SIRT1 siRNA treatment were significantly decreased compared to those in the NAFLD rats treated with only $\mathrm{BP}$, while they were still higher than those in the untreated NAFLD rats (Fig. 5E and F). Moreover, SIRT1 deficiency significantly induced the activity of ROS, which partly suppressed ROS activation via BP in NAFLD rats (Fig. 5G), indicating that $\mathrm{BP}$ inhibited the oxidative stress partly via SIRT1.

\section{BP induced the expression of PGC-1 $\alpha$ via SIRT1}

The mRNA and protein levels of PGC- $1 \alpha$ were significantly decreased in the NAFLD model group compared to the control group (Fig. 6A). In particular, BP restored the mRNA levels of PGC- $1 \alpha$ to normal similar to that in the control group, whereas with silenced SIRT1, the mRNA and protein levels of PGC-1 $\alpha$ were significantly decreased in the NAFLD animals treated with BP (Fig. 6A and B). Similarly, immunohistochemistry 
A

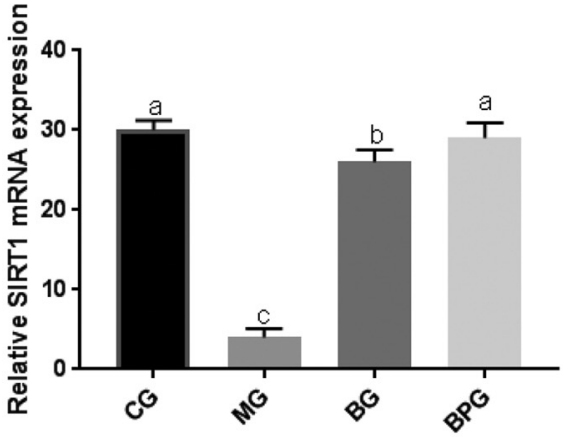

B

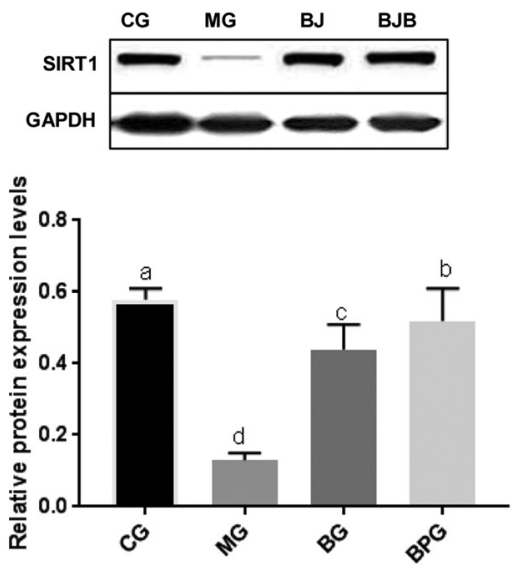

Fig. 3 BP reversed the decrease in the expression of SIRT1 in NAFLD. (A) Expression of SIRT1 was measured by quantitative reverse transcriptionPCR. (B) Expression of SIRT1 was measured by western blot. CG, normal rats; MG, NAFLD model rats; BG, model rats were treated with blueberry juice; BPG, model rats were treated with blueberries and probiotics. Different lowercase letters $(a, b, c$, and d) represent significant differences $(P<$ 0.05 , one way ANOVA, $n=8$ ).

revealed that the PGC-1 $\alpha$ signal was significantly reduced in the NAFLD model group compared to the normal group, while the BP treatment restored the PGC- $1 \alpha$ signal to almost the normal level (Fig. 6C). With SIRT1 deficiency, the effect of BP on the PGC-1 $\alpha$ signal was partly abolished.

\section{Discussion}

Accumulating evidence shows the involvement of blueberry or probiotics in the protection of the liver from diseases due to a high-fat diet ${ }^{17}$ liver fibrosis ${ }^{18}$ and hepatocellular carcinoma. ${ }^{19,20}$ However, little is known about the effect of the combination of blueberry and probiotics on liver diseases. Our previous study revealed that the combination of blueberry juice and probiotics exhibits a better ameliorating effect on Non-Alcoholic Steatohepatitis (NASH) compared to only blueberry or probiotics. ${ }^{10}$ Nevertheless, the molecular mechanisms underlying the effects of BP in NAFLD remain unclear. In the present study, we demonstrated that BP substantially restored the pathogenesis of HFD-induced NAFLD by improving mitochondrial function via activation of the SIRT1/PGC- $1 \alpha$ pathway. Moreover, BP reduced the mitochondrial oxidative stress by suppressing MDA and ROSD, and activating GSH and SOD.

Mitochondrial dysfunction arises in NAFLD and contributes to the progression from simple steatosis to steatohepatitis. ${ }^{21}$ The oxidation of fatty acids induces the production of ROS, which triggers mitochondrial dysfunction, including disruption of the mitochondrial respiratory chain, loss of mitochondrial membrane potential, and damage to mitochondrial structural integrity. In the present study, we found that hepatic mitochondria were swollen and mitochondrial integrity was damaged in HFD-induced NAFLD rats, which was in agreement with the previous study. ${ }^{22}$ Furthermore, impaired mitochondria can lead to a decline in the activities of mitochon- drial respiratory chain complexes in NAFLD rats. ${ }^{23}$ Similarly, our present data showed that BP not only reestablishes mitochondrial size and liver integrity, but also decreases state 4 and 3 respiration rates, increases the RCR and ADP/O ratio, and inhibits the synthesis of ATP, ADP, AMP, and EC. These results indicated that $\mathrm{BP}$ efficiently improved the structure and function of hepatic mitochondria during NAFLD.

Oxidative stress refers to various adverse processes arising due to the imbalance between the excessive formation of ROS and/or reactive nitrogen species and limited antioxidant defenses. ${ }^{24}$ When the mitochondrial function is impaired, mitochondria produce excessive ROS and generate oxidative stress, resulting in NAFLD development. ${ }^{34}$ ROS "attack" polyunsaturated fatty acids, which produce MDA and HNE as the by-products of aldehydes. ${ }^{25}$ However, with the increase of mitochondrial ROS production during NAFLD, the expression and activity of ROS detoxification mechanisms (such as SOD2 and GSH) are decreased, as reported in in vitro and in vivo studies. ${ }^{26}$ In the present study, we showed that BP could significantly enhance the SOD and GSH levels and reduce the MDA contents in rat livers, indicating that BP exhibited antioxidant properties.

Sirtuins (Sirt1-Sirt7) control critical cellular processes in the nucleus, cytoplasm, and mitochondria to maintain metabolic homeostasis, reduce cellular damage, and dampen inflammation. ${ }^{27}$ Regulation of SIRT1 expression is essential to protect the liver from cholestatic liver disease. ${ }^{28}$ Song et al. suggested that metformin alleviates hepatic steatosis through PRKA-independent, SIRT1-mediated effects on the autophagy machinery ${ }^{29}$ Dioscin markedly prevented NAFLD by adjusting lipid metabolism via the SIRT1/AMPK signal pathway, which should be considered as a new therapeutic candidate for NAFLD. ${ }^{30}$ A previous study showed that the expression of SIRT1 is reduced significantly in NAFLD induced by a high-fat diet in rats and CR improves NAFLD by increasing the SIRT1 protein expression. ${ }^{31}$ Similarly, our study indicated that the 
A

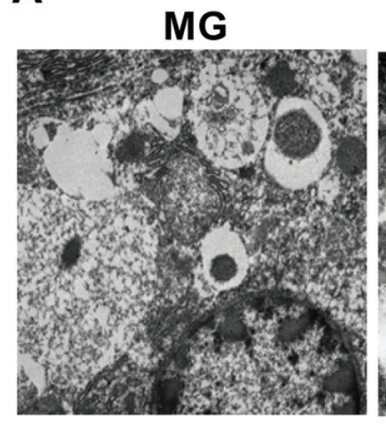

C

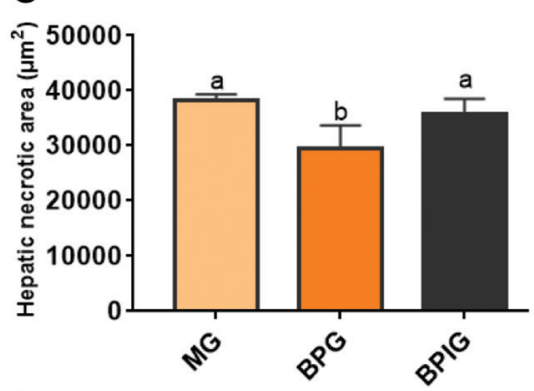

$\mathbf{F} \quad \mathbf{G}$

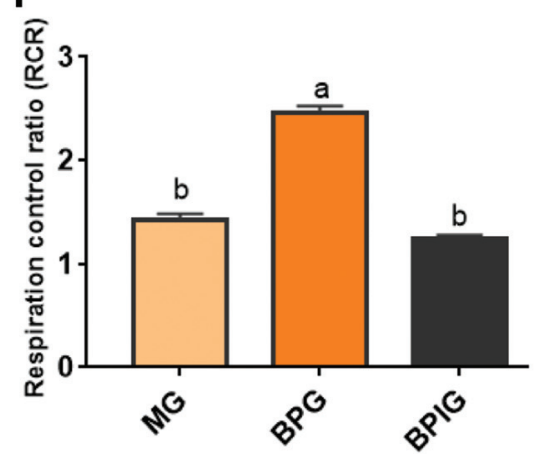

I

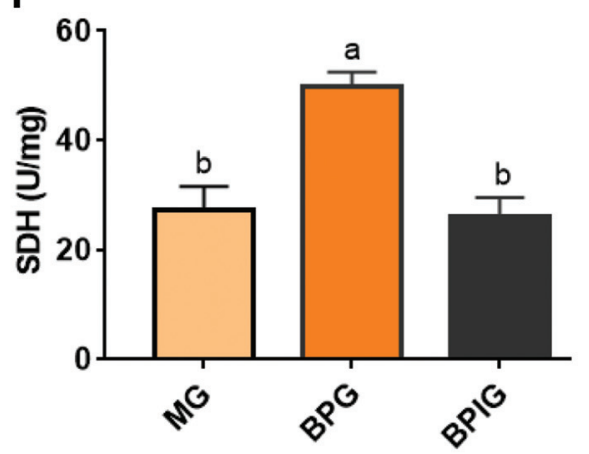

BPG

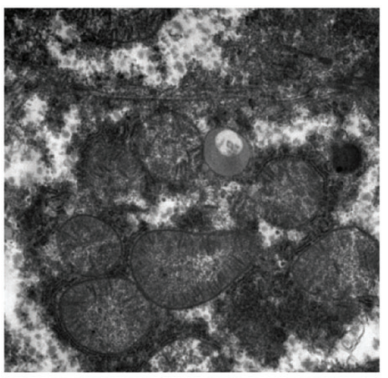

D
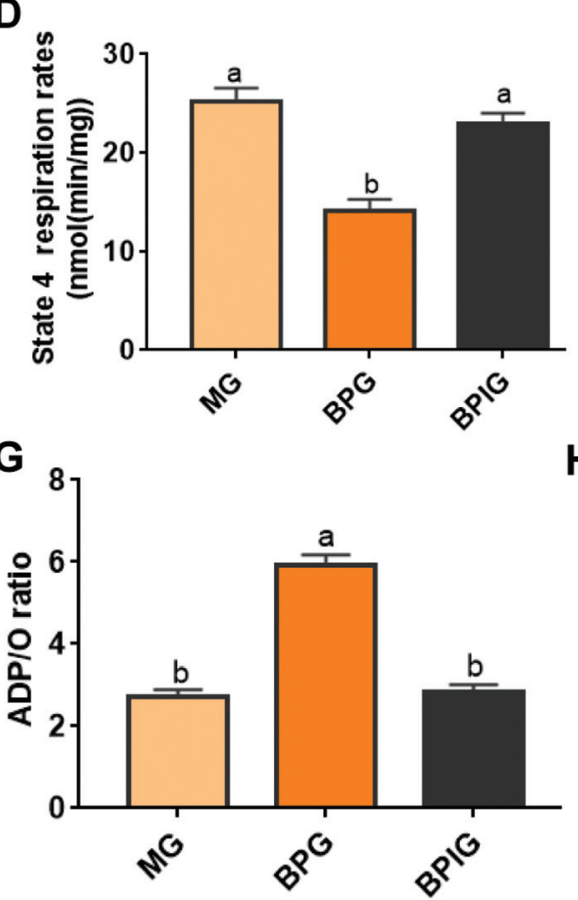

B

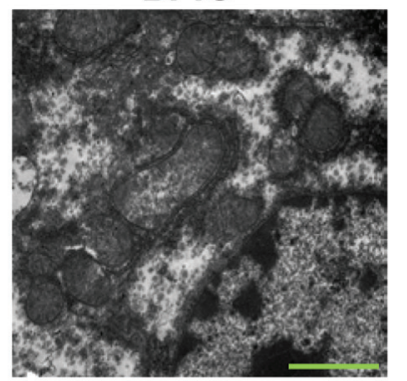

E

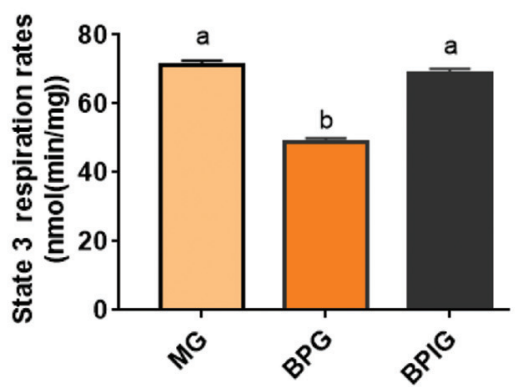

H

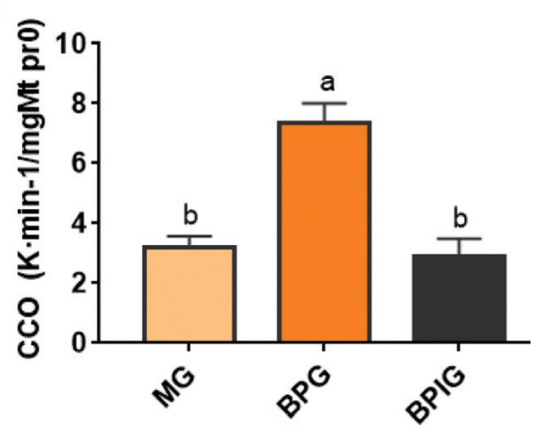

$\mathrm{J}$

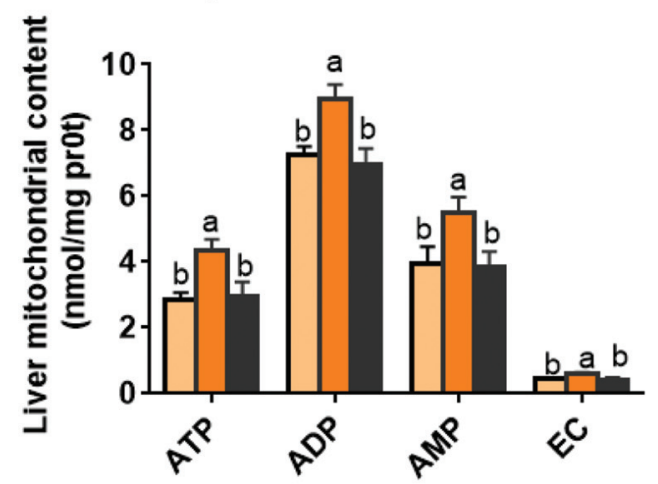

Fig. 4 SIRT1 silencing suppressed the mitochondrial protection by BP. (A) Electron micrographs of mitochondria in different groups. Scale bars: $2 \mu \mathrm{m}$. (B) The degree of mitochondrial swelling was measured by the absorbance value of the hepatic mitochondrial suspension at $520 \mathrm{~nm}$. (C) The area of hepatic necrosis was measured by a section of liver tissue using the Olympus BX41 image system. (D, E, F, and G) Mitochondrial respiratory function was evaluated by state 4 and 3 respiration rates, RCR, and the ADP/O ratio. ( $H$ and I) Mitochondrial function biomarkers were detected using a biochemical marker kit. (J) Mitochondrial synthesis functions of ATP, ADP, AMP, and EC. The MG group was treated with siRNA NC, and BG and BPG groups were treated with SIRT1 siRNA. Different lowercase letters $(a, b, c$, and d) represent significant differences $(P<0.05$, one way ANOVA, $n=8$ ).

mRNA and protein levels of SIRT1 were significantly decreased in the NAFLD group compared to the normal murine. Moreover, SIRT1 deficiency significantly abolished the role of
$\mathrm{BP}$ in mitochondrial protection and anti-oxidative stress, suggesting that BP attenuates the mitochondrial function and oxidative stress via SIRT1. 
A

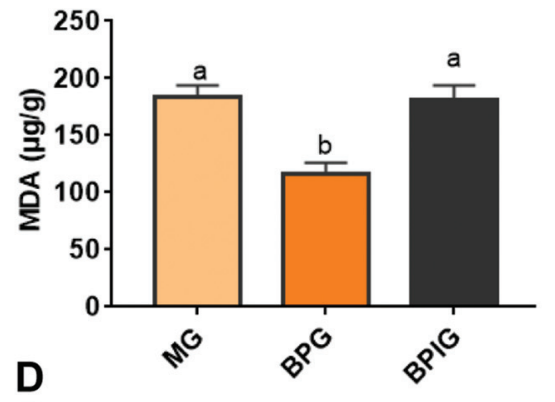

B

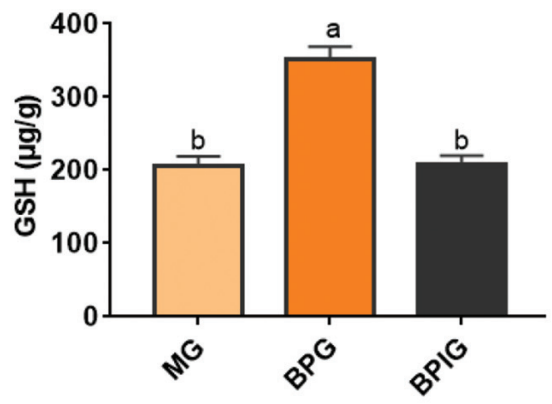

E
C

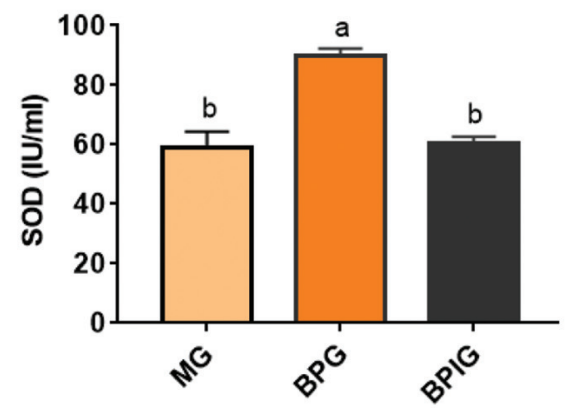

$\mathbf{F}$

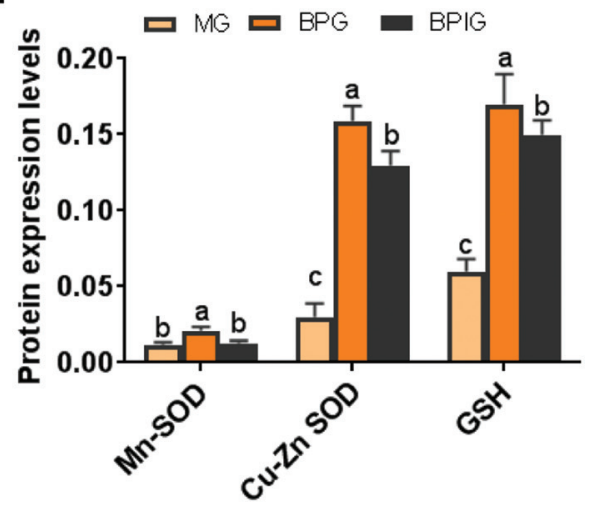

G

MG

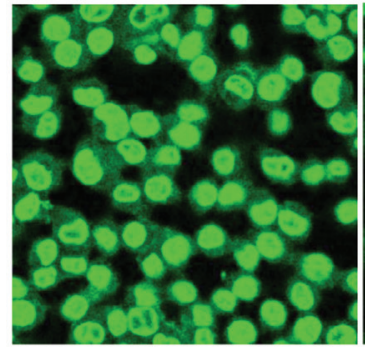

BPG

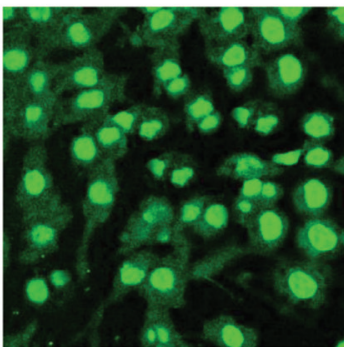

BPIG

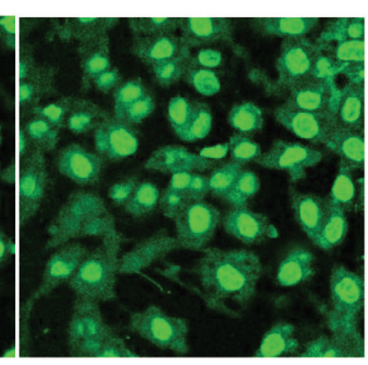

H

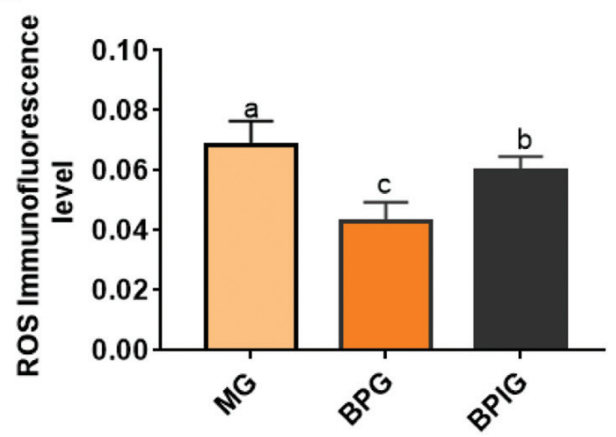

Fig. 5 SIRT1 silencing abolished the anti-oxidative stress function of BP. (A, B, and C) Oxidative stress biomarkers were detected by using a biochemical marker kit. (D, E and F) Expression of SOD and GSH was measured by quantitative reverse transcription-PCR and western blot. (G and H) Reactive oxygen species (ROS) in liver were detected by immunofluorescence ( $\times 400)$. The MG group was treated with siRNA NC, and BG and BPG groups were treated with SIRT1 siRNA. Different lowercase letters $(a, b, c$, and d) represent significant differences $(P<0.05$, one way ANOVA, $n=8)$.

Numerous studies have revealed that PGC1 $\alpha$ plays an important role in the mitochondrial function. For instance, IL-15 regenerates mitochondrial fitness by facilitating cell division via restoration of the expression of PGC1 $\alpha .{ }^{32}$ Decreased mitochondrial function and increased ROS are associated with the downregulation of PGC1A in Fibulin-4 mutant mice with aneurysmal aortas. ${ }^{33}$ Melatonin attenuates the development of diabetes-induced cardiac dysfunction by preventing mitochondrial fission via the SIRT1-PGC1 $\alpha$ pathway. ${ }^{34}$ Our study showed that BP significantly increased the expression of PGC1 $\alpha$, while SIRT1 silencing reduced the PGC1 $\alpha$ level and BP function, indicating that the effects of BP on mitochondria and oxidative stress might be mediated by SIRT1-mediated PGC1 $\alpha$ regulation.

In conclusion, this study showed that BP exhibits synergistic effects for preventing the development of NAFLD by protecting mitochondrial function, by suppressing the damage of the mitochondrial ultrastructure, by reducing mitochondrial swelling, and by enhancing the respiration function. Moreover, BP ameliorated HFD-induced liver damage via the reduction of oxidative stress by reducing the ROS activity and MDA level, and increasing the GSH and SOD levels. SIRT1 deficiency sig- 

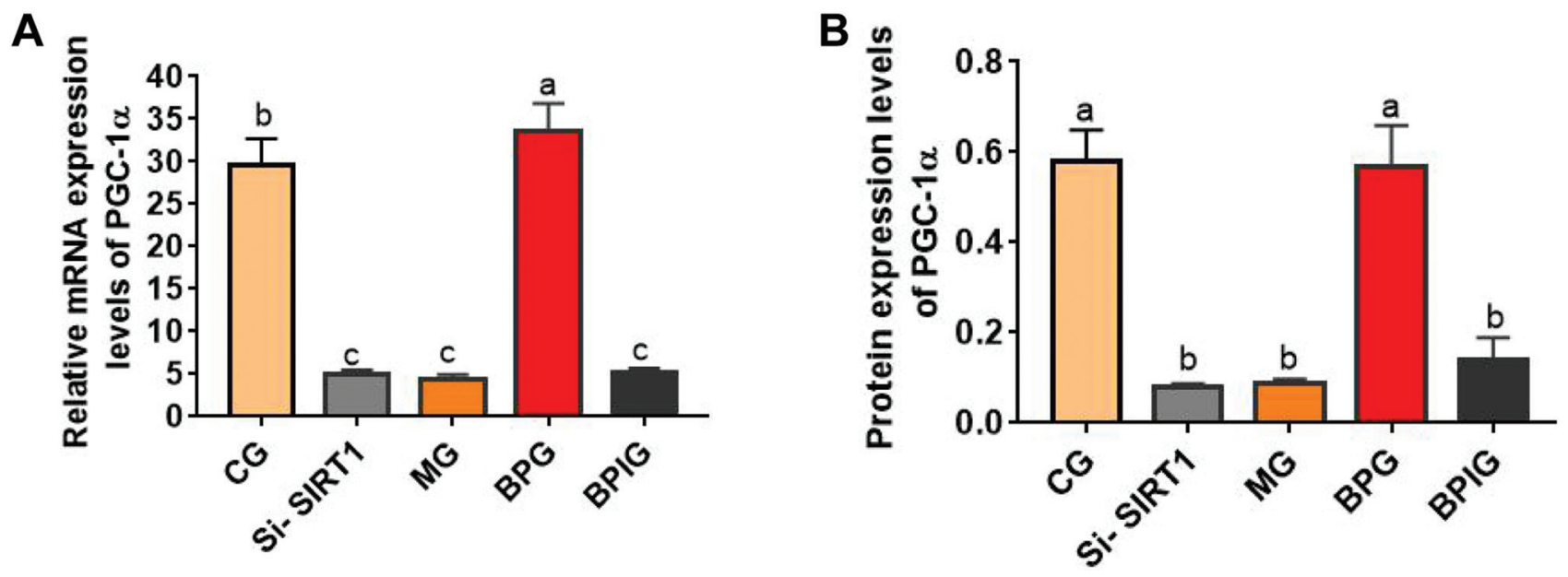

C

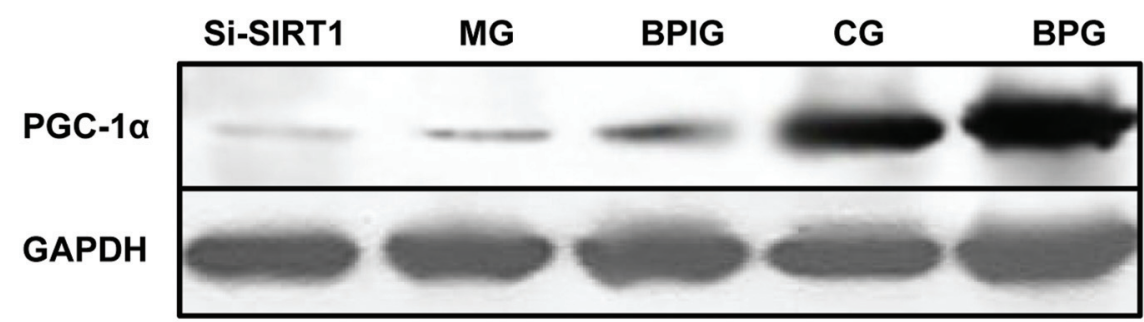

D

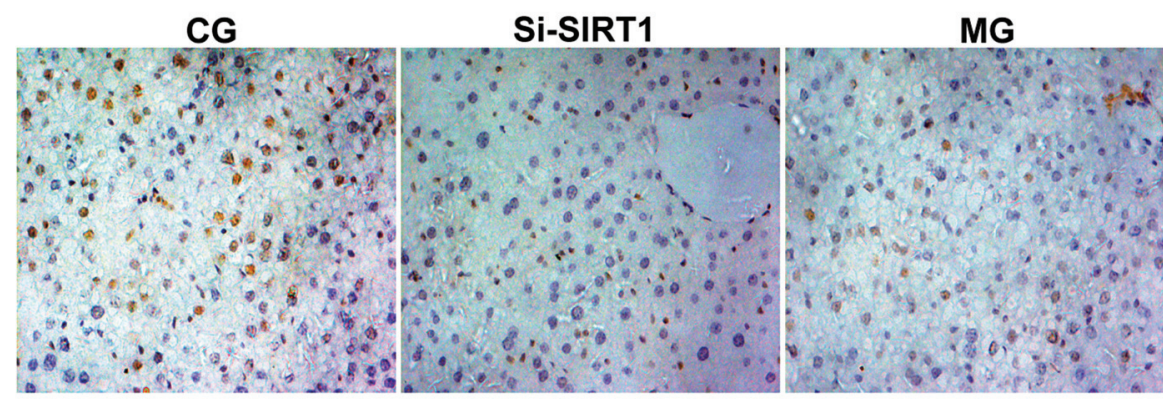

BPG

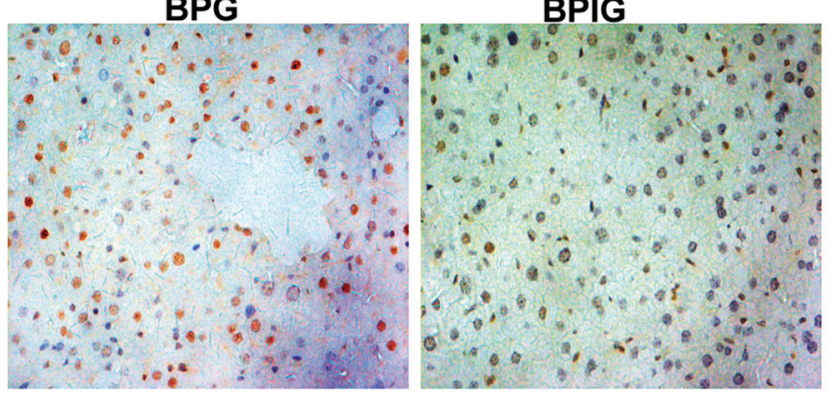

Fig. 6 SIRT1 silencing reversed BP-mediated downregulation of PGC-1 $\alpha$ in NAFLD rats. (A) Quantitative reverse transcription-PCR analysis of PGC-1 $\alpha$ mRNA expression in each group. Samples were normalized to GAPDH mRNA. (B and C) Western blot of PGC-1 $\alpha$. (D) Immunohistochemical analysis of PGC-1 $\alpha$ in each group $(\times 400)$. Different lowercase letters $(a, b, c, d$, and e) represent significant differences $(P<0.05$, one way ANOVA, $n$ $=8$ ).

nificantly abolished the effects of BP. SIRT1 silencing blocked the BP-induced activation of PGC- $1 \alpha$. Therefore, BP protected mitochondria and inhibited oxidative stress during NAFLD by affecting the SIRT1/PGC-1 $\alpha$ pathway. BP might be developed as food additives for preventing the progression of NAFLD.

\section{Data accessibility}

The datasets used and analyzed during the current study are available from the corresponding author on reasonable request. 


\section{Author contributions}

Conceptualization: Hongchao Feng; data curation: Tingting Ren, Lili Zhu, and Yanyan Shen; investigation: Lili Zhu, Yanyan Shen, Qiuju Mou, and Tao Lin; preparation of the original draft: Tingting Ren.

\section{Conflicts of interest}

The authors declare that they have no competing interests.

\section{Funding}

This work was supported by the National Natural Science Foundation of China (81560100).

\section{Acknowledgements}

We would like to thank all participants involved in this study.

\section{References}

1 A. Cano, et al., Hepatic VLDL assembly is disturbed in a rat model of nonalcoholic fatty liver disease: is there a role for dietary coenzyme Q?, J. Appl. Physiol., 2009, 107(3), 707717.

2 P. Angulo, Nonalcoholic fatty liver disease, Rev. Gastroenterol. Mex, 2005, 70(Suppl. 3), 52-56.

3 M. R. Teli, et al., Determinants of progression to cirrhosis or fibrosis in pure alcoholic fatty liver, Lancet, 1995, 346(8981), 987-990.

4 N. Stefan, H. U. Haring and K. Cusi, Non-alcoholic fatty liver disease: causes, diagnosis, cardiometabolic consequences, and treatment strategies, Lancet Diabetes Endocrinol., 2018, DOI: 10.1016/S2213-8587(18)30154-2.

5 A. Borrelli, et al., Role of gut microbiota and oxidative stress in the progression of non-alcoholic fatty liver disease to hepatocarcinoma: Current and innovative therapeutic approaches, Redox Biol., 2018, 15, 467-479.

6 M. G. Zhao, et al., Triterpenic acids-enriched fraction from Cyclocarya paliurus attenuates non-alcoholic fatty liver disease via improving oxidative stress and mitochondrial dysfunction, Biomed. Pharmacother., 2018, 104, 229-239.

7 S. Skrovankova, et al., Bioactive Compounds and Antioxidant Activity in Different Types of Berries, Int. J. Mol. Sci., 2015, 16(10), 24673-24706.

8 L. Ma, et al., Molecular Mechanism and Health Role of Functional Ingredients in Blueberry for Chronic Disease in Human Beings, Int. J. Mol. Sci., 2018, 19(9), 2785.

9 L. Xue, et al., Probiotics may delay the progression of nonalcoholic fatty liver disease by restoring the gut microbiota structure and improving intestinal endotoxemia, Sci. Rep., 2017, 7, 45176.
10 T. Ren, et al., The Combination of Blueberry Juice and Probiotics Ameliorate Non-Alcoholic Steatohepatitis (NASH) by Affecting SREBP-1c/PNPLA-3 Pathway via PPARalpha, Nutrients, 2017, 9(3), pii: E198.

11 A. Brunet, et al., Stress-dependent regulation of FOXO transcription factors by the SIRT1 deacetylase, Science, 2004, 303(5666), 2011-2015.

12 R. B. Ding, J. Bao and C. X. Deng, Emerging roles of SIRT1 in fatty liver diseases, Int. J. Biol. Sci., 2017, 13(7), 852-867.

13 M. B. Hock and A. Kralli, Transcriptional control of mitochondrial biogenesis and function, Annu. Rev. Physiol., 2009, 71, 177-203.

14 M. Rossi, et al., Effect of fruit blanching on phenolics and radical scavenging activity of highbush blueberry juice, Food Res. Int., 2003, 36(9), 999-1005.

15 G. Q. Wen, F. Nie and Y. J. Liao, Comparative analysis of physicochemical component content in blueberry fruit and their function evaluation, Acta Agric. Jiangxi, 2012, 24(1), 117-119.

$16 \mathrm{~J}$. Zhu, et al., Blueberry, combined with probiotics, alleviates non-alcoholic fatty liver disease via IL-22-mediated JAK1/STAT3/BAX signaling, Food Funct., 2018, 9(12), 62986306.

17 S. Lee, et al., Blueberry Supplementation Influences the Gut Microbiota, Inflammation, and Insulin Resistance in High-Fat-Diet-Fed Rats, J. Nutr., 2018, 148(2), 209-219.

18 Y. Wang, et al., Dietary supplementation of blueberry juice enhances hepatic expression of metallothionein and attenuates liver fibrosis in rats, PLoS One, 2013, 8(3), e58659.

19 W. Zhan, et al., Effects of blueberries on migration, invasion, proliferation, the cell cycle and apoptosis in hepatocellular carcinoma cells, Biomed. Rep., 2016, 5(5), 579-584.

$20 \mathrm{~J}$. Li, et al., Probiotics modulated gut microbiota suppresses hepatocellular carcinoma growth in mice, Proc. Natl. Acad. Sci. U. S. A., 2016, 113(9), E1306-E1315.

21 I. Grattagliano, et al., Targeting mitochondria to oppose the progression of nonalcoholic fatty liver disease, Biochem. Pharmacol., 2018, 160, 34-45.

22 I. C. M. Simoes, et al., Mitochondria in non-alcoholic fatty liver disease, Int. J. Biochem. Cell Biol., 2018, 95, 93-99.

23 A. Mansouri, C. H. Gattolliat and T. Asselah, Mitochondrial Dysfunction and Signaling in Chronic Liver Diseases, Gastroenterology, 2018, 155(3), 629-647.

24 A. P. Rolo, J. S. Teodoro and C. M. Palmeira, Role of oxidative stress in the pathogenesis of nonalcoholic steatohepatitis, Free Radicals Biol. Med., 2012, 52(1), 59-69.

$25 \mathrm{X}$. Yin, et al., Glucose fluctuation increased hepatocyte apoptosis under lipotoxicity and the involvement of mitochondrial permeability transition opening, J. Mol. Endocrinol., 2015, 55(3), 169-181.

26 A. Besse-Patin, et al., Estrogen Signals Through Peroxisome Proliferator-Activated Receptor-gamma Coactivator 1alpha to Reduce Oxidative Damage Associated With Diet-Induced Fatty Liver Disease, Gastroenterology, 2017, 152(1), 243256. 
27 S. Winnik, et al., Protective effects of sirtuins in cardiovascular diseases: from bench to bedside, Eur. Heart J., 2015, 36(48), 3404-3412.

28 B. A. Blokker, et al., Fine-tuning of SIRT1 expression is essential to protect the liver from cholestatic liver disease, Hepatology, 2018, 69(2), 699-716.

29 Y. M. Song, et al., Metformin alleviates hepatosteatosis by restoring SIRT1-mediated autophagy induction via an AMPactivated protein kinase-independent pathway, Autophagy, 2015, 11(1), 46-59.

$30 \mathrm{H}$. Yao, et al., Dioscin alleviates non-alcoholic fatty liver disease through adjusting lipid metabolism via SIRT1/AMPK signaling pathway, Pharmacol. Res., 2018, 131, 51-60.
31 X. Q. Deng, L. L. Chen and N. X. Li, The expression of SIRT1 in nonalcoholic fatty liver disease induced by highfat diet in rats, Liver Int., 2007, 27(5), 708-715.

32 S. A. Younes, et al., Cycling CD4+ T cells in HIV-infected immune nonresponders have mitochondrial dysfunction, J. Clin. Invest., 2018, 128(11), 5083-5094.

33 I. van der Pluijm, et al., Decreased mitochondrial respiration in aneurysmal aortas of Fibulin-4 mutant mice is linked to PGC1A regulation, Cardiovasc. Res., 2018, 114(13), 1776-1793.

34 M. Ding, et al., Melatonin prevents Drp1-mediated mitochondrial fission in diabetic hearts through SIRT1PGC1alpha pathway, J. Pineal Res., 2018, 65(2), e12491. 